\title{
Representação do conhecimento incerto aplicado a sistemas educacionais na $\mathrm{Web}^{1}$
}

\section{Uncertain knowledge representation applied to educational system in Web}

\author{
Rosa MariaVicari* \\ Cecília Dias Flores** \\ Marcos Eduardo Casa*** \\ Alexandre Moretto Ribeiro ${ }^{* * * *}$
}

\begin{abstract}
RESUMO
Este artigo apresenta os resultados obtidos no projeto conjunto entre o Instituto de Informática da UFRGS (II-UFRGS) e o Departamento de Informática da UCS (DEIN/UCS), cujo propósito foi a proposição e o desenvolvimento de um ambiente educacional multiagentes, baseado na web. $\mathrm{O}$ foco principal da pesquisa está no desenvolvimento de um modelo de representação do conhecimento para a construção de agentes inteligentes, capazes
\end{abstract}

1 Este projeto teve apoio dos seguintes órgãos de fomento à pesquisa: Fundação de Amparo à Pesquisa do Rio Grande do Sul - Fapergs - e Conselho Nacional de Pesquisa CNPq -, através do Programa Sul de Pesquisa e Pós Graduação - PSPPG.

* Ph.D. em Engenharia Eletrotécnica e Computadores pela Universidade de Coimbra/Portugal. Professora do Instituto de Informática da Universidade Federal do Rio Grande do Sul.rosa@inf.ufrgs.br

** Doutoranda em Ciência da Computação na UFRgs. Professora do Instituto de Informática da Universidade Federal do Rio Grande do Sul (II-UFRGS). dflores@inf.ufrgs.br

*** Ph.D. em Ciência da Computação pela University of Birmingham - Inglaterra. Professor do Departamento de Informática da Universidade de Caxias do Sul (DEIN-UCS). mecasa@ucs.br

**** Ph.D. em Informática pela Université de Grenoble I (Scientifique Et Medicale Joseph Fourier), Grenoble/ França. Professor Titular do Departamento de Informática da Universidade de Caxias do Sul (DEIN -UCS). aribeiro@ucs.br 
de atuar em situações reais de ensino e aprendizagem. Em particular, o foco está no uso de métodos probabilísticos de representação do conhecimento, para a realização de diagnósticos clínicos.

Palavras-chave: informática na educação, inteligência artificial, representação do conhecimento.

\begin{abstract}
This paper presents some results about a join Project between Instituto de Informática da UFRGS (II-UFRGS) and Departamento de Informática da UCS (DEIN/UCS), that proposed and implemented an educational multi-agent environment based at web. The research focus is on the knowledge representation applied to the construction of intelligent agents able to act in educational real environments. More specifically, the work aims at applying probabilistic knowledge representation in the aid to medical diagnostic in educational applications.

Key-words: computer and education, artificial intelligence, knowledge representation.
\end{abstract}

\title{
Introdução
}

O emprego de técnicas de inteligência artificial para a construção de agentes inteligentes, em ambientes computacionais, é uma área que vem sendo utilizada para o desenvolvimento de sistemas computacionais de aprendizagem. Um problema clássico da área é a proposição de modelos de representação do conhecimento, adequados para a construção de bases de conhecimento, capazes de dar suporte à atuação desses agentes artificiais.

As pessoas resolvem problemas e tomam decisões em ambientes onde a informação é parcial (isto é, não completa) ou aproximada (isto é, não exata). Têm-se tentado emular essa capacidade em agentes inteligentes, mas para problemas formulados com informação parcial ou aproximada pode-se obter apenas soluções aproximadas, ou seja, com incerteza. Portanto, torna-se necessário dispor de formas para lidar com a incerteza.

O projeto Pesquisa e Aplicação de Métodos de Representação do Conhecimento, desenvolvido entre o Instituto de Informática da UFRGS (II-UFRGS) e o Departamento de Informática da UCS (DEIN/UCS), teve por 
objetivo propor e implementar Modelos de Representação do Conhecimento, aplicados à construção de Agentes Inteligentes capazes de atuar em ambientes reais. Mais especificamente estudou-se modelos de representação do conhecimento, aplicados em ambientes de auxílio ao diagnóstico médico, com fins educacionais. Estas áreas são exemplos de ambientes formados por conhecimento incerto.

Com a construção desta infra-estrutura, o objetivo é produzir Ambientes de Aprendizagem onde múltiplas formas externas (fora da máquina) de representação, utilizadas nos processos de representação do conhecimento e resolução de problemas, estejam disponíveis. Desta maneira, o aluno tem maior liberdade para explorar problemas de diversos pontos de vista. Neste contexto, Agentes Inteligentes podem monitorar o processo de interação com o ambiente e proporcionar auxílio ao aluno. Ou seja, o Ambiente oferece uma interface flexível, que abre possibilidades para adaptar o estilo de interação do ambiente aos diferentes estilos cognitivos (por exemplo, lingüístico versus espacial), preferências e situações do aluno. A estrutura do Ambiente Inteligente, a aparência das interfaces oferecidas e o estilo de interação com o sistema são definidos de acordo com observações realizadas pelos Agentes Inteligentes.

Este artigo está organizado da seguinte forma: na próxima seção apresenta-se a plataforma para construção de ambientes de aprendizagem PMA $^{3}$ (Plataforma Multiagentes para Ambientes de Aprendizagem) que está sendo desenvolvida pelo DEIN/UCS; na seção seguinte, apresenta-se o método de representação do conhecimento (redes bayesianas), empregado no desenvolvimento do primeiro ambiente de aprendizagem, que foi implementado sobre a plataforma $\mathrm{PMA}^{3}$; na quarta seção, descreve-se o projeto Amplia (Ambiente Multiagente Probabilístico Inteligente de Aprendizagem), que está sendo desenvolvido pelo II-UFRGS; e, por último, apresentam-se algumas considerações finais sobre esse projeto conjunto.

\section{Plataforma para construção de Ambientes de Aprendizagem}

Visando explorar as potencialidades dos Sistemas Multiagentes (SMA), foi desenvolvida uma plataforma para construção de Ambientes de Aprendizagem, denominada $\mathrm{PMA}^{3}$. $\mathrm{APMA}^{3}$ foi desenvolvida sobre a infraestrutura 
disponível no ambiente FIPA-OS (desenvolvido e distribuído pela Nortel Networks Harlow Laboratories) que implementa uma arquitetura específica para Sistemas Multiagentes, baseado nas especificações do padrão Fipa (Foundation for Intelligent Physical Agents).

De acordo com o modelo de aprendizagem adotado, disponibilizamos na $\mathrm{PMA}^{3}$ um conjunto de serviços para o gerenciamento do ambiente e serviços, visando facilitar a comunicação entre os diversos participantes do processo de aprendizagem. O objetivo é construir ambientes específicos de aprendizagem onde o estudante tenha autonomia e iniciativa na condução do seu próprio processo de aprendizagem. Por outro lado, o facilitador da aprendizagem (professor e/ou monitores) dispõem de recursos para organizar atividades de aprendizagem e acompanhar o trabalho dos estudantes.

O princípio de construção dessa plataforma para ambientes de aprendizagem é, portanto, a interação. Uma das principais contribuições da utilização de SMA em ambientes de aprendizagem é promover a interação entre os diversos atores do sistema, sejam eles humanos ou artificiais. Além disto, a plataforma deve prever os recursos necessários para que o processo de interação possa ser acompanhado.

A arquitetura proposta para a construção de Ambientes de Aprendizagem, baseados em SMA, está organizada em três camadas (ver figura 1): na base encontra-se uma plataforma multiagentes, na camada intermediária a plataforma multiagentes é estendida com novos serviços, gerando a $\mathrm{PMA}^{3}$ e na terceira camada encontram-se os ambientes de aprendizagem propriamente ditos.

FIGURA 1 - ARQUITETURA PARA AMBIENTES DE APRENDIZAGEM BASEADOS EM SMA

\begin{tabular}{|c|c|c|c|}
\hline $\begin{array}{c}\text { Ambiente de } \\
\text { Aprendizagem 1 }\end{array}$ & $\begin{array}{c}\text { Ambiente de } \\
\text { Aprendizagem 2 }\end{array}$ & $\cdots$ & $\begin{array}{c}\text { Ambiente de } \\
\text { Aprendizagem } n\end{array}$ \\
\hline PMA $^{3}$ & \\
\hline \multicolumn{3}{|c|}{ Plataforma SMA } \\
\hline
\end{tabular}

Para a primeira camada, foi escolhida a plataforma multiagentes FIPAOS, desenvolvida pela Nortel Networks (FIPA-OS, 2001). Esta camada oferece o serviço de comunicação entre agentes, o serviço de gerenciamento do sistema e o serviço de diretórios. 
A plataforma multiagentes deve ser genérica o suficiente para que possa ser utilizada no desenvolvimento de diversos ambientes de aprendizagem. Com isto em mente, foi escolhida uma plataforma que levasse em consideração os padrões propostos pela FIPA, no que concerne às tecnologias relacionadas a agentes. Desta forma, será possível agregar novos agentes, desde que compatíveis com os padrões FIPA, independentemente de onde forem desenvolvidos.

Na segunda camada da $\mathrm{PMA}^{3}$ estão sendo portados alguns dos serviços propostos como básicos para o Modelo de Interação Dinâmico (RIBEIRO; DEMAZEAU, 2001). Estes serviços devem complementar aqueles propostos pela Fipa, e que se encontram disponíveis no FIPA-OS. Os serviços selecionados são: o Serviço de organização do sistema, que fornece um mecanismo para a representação explícita dos aspectos estruturais da organização do SMA (FERBER; GUTKNECHT, 1998), os serviços de interação entre usuários (comunicação síncrona e assíncrona), o gerenciamento de problemas e a navegação colaborativa. Com isto, pretende-se levar em conta os aspectos sociais do processo de aprendizagem, onde a interação entre os estudantes e estudantes e professores tem um papel fundamental (ANDRADE et al., 2001).

$\mathrm{Na}$ figura 2 pode ser observada a interface para o cadastramento de grupos do SOS.

FIGURA 2 - INTERFACES PARA CADASTRO DE GRUPOS

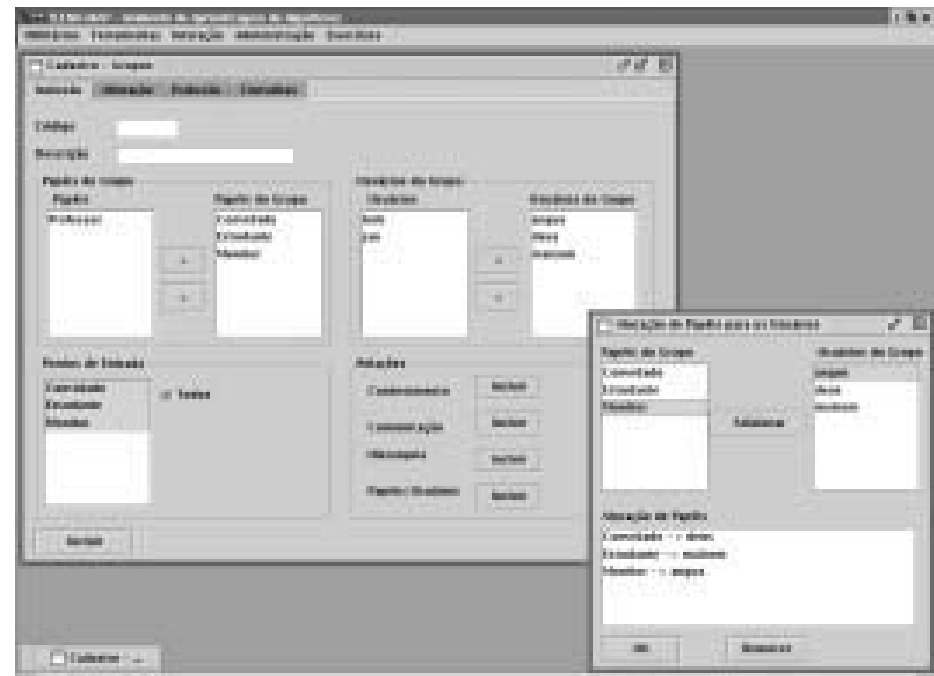


Também faz parte da segunda camada o serviço de registro, que documenta as ações realizadas no ambiente. Além disto, ficam nesta camada os agentes que irão representar os estudantes e professores, e que tem como principal função auxiliar na interação entre os agentes humanos e artificiais presentes no ambiente de aprendizagem.

$\mathrm{Na}$ terceira camada estão os componentes específicos de cada ambiente de aprendizagem. No caso do Amplia (VICARI, 2003), é nesta camada que devem entrar os agentes que o compõem, bem como outros agentes voltados ao acompanhamento das atividades desempenhadas por seus usuários.

\section{Modelo de Representação do Conhecimento Incerto}

No final dos anos 80, houve uma retomada de interesse por abordagens probabilísticas para o tratamento da incerteza, motivada pela descoberta de que, considerando o relacionamento causal e a independência (condicional) entre variáveis do domínio, é necessário representar apenas as probabilidades condicionais entre variáveis diretamente dependentes (e não mais entre todas as variáveis), tornando essa representação tratável em computador. Essa retomada está associada ao aparecimento das redes probabilísticas, isto é, modelos baseados em representações gráficas das dependências probabilísticas do domínio da aplicação. Redes bayesianas (BN), redes de Markov (MN) e diagramas de influências (ID) são redes probabilísticas.

Uma BN é um grafo acíclico orientado, onde os nós representam variáveis aleatórias e o arco unindo dois nós representa a dependência probabilística entre as variáveis associadas. Cada nó armazena a função de distribuição de probabilidades condicional dos valores que podem ser assumidos pela variável aleatória associada ao nó, dados os valores de seus nós pais (isto é, aqueles diretamente ligados ao nó em questão). A partir de uma BN pode-se construir uma MN equivalente (grafo acíclico similar, mas que não possui arcos orientados). Ambas são representações compactas da função de distribuição de probabilidades conjunta global, de todas as variáveis aleatórias do domínio modelado. 
A característica principal das redes probabilísticas é a habilidade para explorar a estrutura do grafo e reduzir o cálculo (da probabilidade condicional de um evento, dada a evidência disponível) a uma série de cálculos locais, usando somente variáveis obtidas de um nó e seus vizinhos, em uma estrutura de grafo, evitando calcular a função de distribuição de probabilidades conjunta global. A representação gráfica também explicita relações de dependência e constitui uma ferramenta poderosa na aquisição de conhecimentos e no processo de verificação (FLORES et al., 2001).

Para o aumento de performance do cálculo de probabilidade condicional de cada nó do grafo, bem como forma de resolução do problema de cálculo de redes multiconectadas (que podem gerar resultados de crença errôneos em nós convergentes de caminhos múltiplos originários de um nó fonte único) foi proposto o método de universo de crenças por Jensen (JENSEN; OLSEN; ANDERSEN, 1990). Este método transforma a topologia de uma $\mathrm{BN}$ em uma árvore de junção.

O projeto Amplia utiliza BN para a representação do conhecimento do domínio, fazendo uso do método de transformação topológica, proposto por Jensen, para calcular as probabilidades condicionais.

\section{Amplia e sua arquitetura}

O Amplia é um ambiente inteligente de aprendizagem (situado na terceira camada da $\mathrm{PMA}^{3}$ ), projetado como um recurso adicional para a formação dos estudantes de Medicina. Tem como objetivo apoiar o desenvolvimento do raciocínio diagnóstico e a modelagem das hipóteses diagnósticas na área médica (VICARI, 2003).

A proposta é que o aluno gere uma BN para criar a sua representação do conhecimento, envolvendo aspectos qualitativos e quantitativos, a partir de um determinado problema apresentado para estudo. O modelo de rede criado pelo aluno será comparado ao do especialista e as diferenças entre eles serão tratadas através de estratégias pedagógicas adequadas a cada caso. O objetivo é possibilitar ao aluno a visualização da organização das suas idéias, elaborar e testar hipóteses e as reavaliar constantemente, interagindo diretamente com a sua representação do conhecimento, manipulando-o e observando as conseqüências de suas intervenções. 
As figuras 3 e 4 apresentam respectivamente uma rede sobre o diagnóstico da Dengue, modelada por um especialista e uma rede criada por um aluno hipotético.

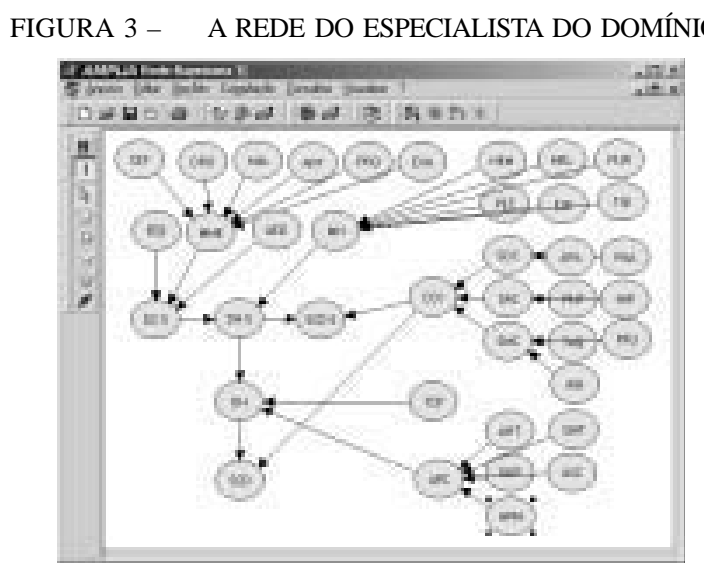

FIGURA 4 - A REDE HIPOTÉTICA DO ALUNO

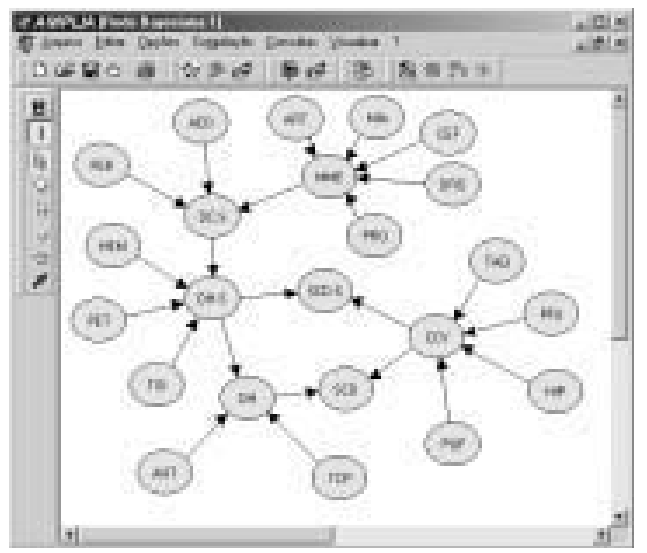

A ferramenta gráfica, denominada Seamed (FLORES et al., 2001), possibilita tanto a construção da rede com seus nós (variáveis) e arcos (relações de dependência probabilística), como a consulta, através da entrada de uma evidência que será propagada através das demais variáveis, com a atualização das probabilidades condicionais, dada esta evidência. O Amplia possui a seguinte arquitetura, conforme a figura 5 . 
O Agente Aprendiz representa o aluno no ambiente multiagente, ou seja, é através do Agente Aprendiz que o aluno interage com o Amplia. Esta representação do aluno possui ainda informações, como nome do aluno, login e senha de acesso, modelo de rede construído e sua auto-avaliação; informações extraídas da grade curricular (posicionamento e desempenho no curso) e registro do $\log$ das ações do aluno, durante a modelagem de sua rede hipotética. O modelo do aluno é continuamente atualizado pelo Agente Aprendiz, que possui, assim, a representação virtual do aluno no ambiente. Em termos da Negociação Pedagógica (FLORES et al., 2002), o Agente Aprendiz representa o aluno, reunindo todas as evidências concretas sobre o estado do seu processo de aprendizagem, registrando seu grau de autoconfiança declarado e buscando inferir a sua confiança em relação aos demais agentes do sistema.

FIGURA 5 - $\quad$ ARQUITETURA DO AMPLIA

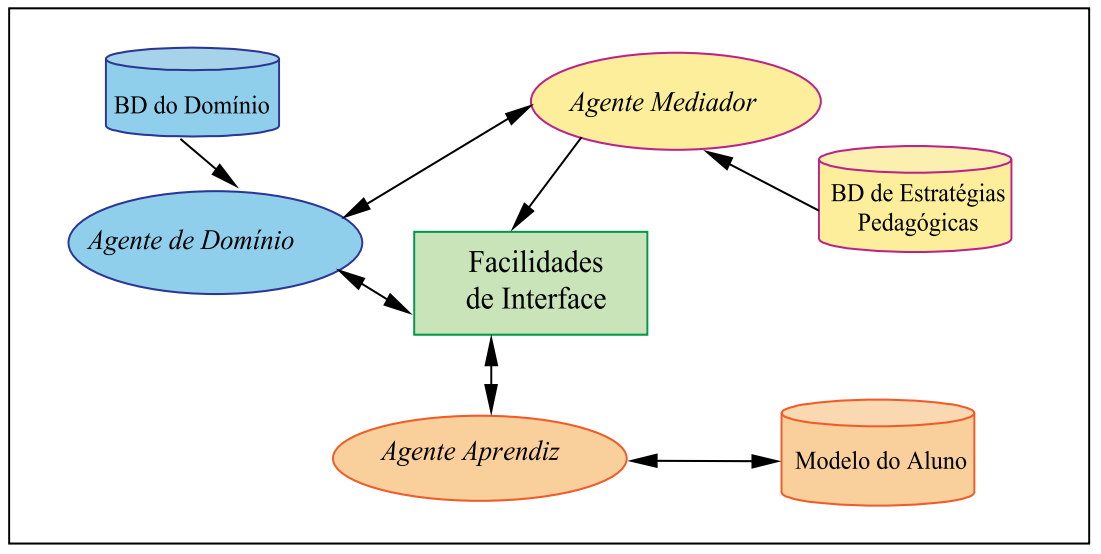

Agente de Domínio compara a rede modelada pelo especialista com o modelo construído pelo aluno, identificando os pontos de conflito. O resultado desta análise é encaminhado para o Agente Mediador. O Agente de Domínio tem acesso à Base de Dados do Domínio que contém a classificação dos nós, os recursos de explanação fornecidos pelo especialista (sob a forma de argumentos), um texto sobre o problema modelado (o estudo de caso) e uma relação de nós para o estudo de caso.

O Agente Mediador toma decisões sobre como e quando intervir no processo de construção do modelo de rede do aluno, após ter identificado um conflito ou impasse (por exemplo, quando há erros de construção da 
rede ou quando a rede é inviável), mas também pode agir mediante uma solicitação do aluno. A função principal do Agente Mediador, entretanto, é selecionar a estratégia pedagógica mais apropriada, possibilitando a resolução de conflitos que podem ocorrer entre os Agentes de Domínio e Aprendiz.

Os Agentes de Domínio e Mediador compartilham o papel do professor. O Agente de Domínio incorpora a base de conhecimentos sobre o tema a ser estudado e, portanto, tem o maior grau de confiança sobre o assunto. É ele o responsável pelos conteúdos e pela avaliação do progresso do aluno. O Agente Mediador incorpora os mecanismos de negociação necessários para resolver os conflitos neste processo, ou seja, as estratégias pedagógicas de ensino que podem ser usadas na negociação pedagógica.

\section{O papel da negociação pedagógica}

A idéia básica do papel da negociação pedagógica é que, embora o processo de ensino-aprendizagem possa ser considerado um processo de equalização de graus de confiança, não se pode pressupor que ele tenha um comportamento linear, monotônico e crescente. Ou seja, não se pode assegurar que este processo irá aumentar um determinado grau de confiança passo-a-passo (linear), sem interrupções (monotônico), nem retrocessos (crescente), até atingir o nível desejado. É possível que ocorram conflitos de avaliações do professor em relação ao aluno, e vice-versa, sendo então necessário acionar o processo de negociação pedagógica para, dentro do possível, resolver o conflito através de mecanismos de argumentação, com o propósito de reforçar a relação de confiança entre o par aluno/ professor. Ou seja, o resultado esperado de um processo de negociação pedagógica é o aumento do grau de confiança entre os agentes que estão interagindo nesta negociação.

Um outro aspecto importante na definição de um mecanismo de negociação pedagógica é a definição de qual estratégia de ensino-aprendizagem deve ser utilizada. Este é um assunto que envolve essencialmente a decisão de qual escola de ensino-aprendizagem será adotada pelo sistema (comportamentalista, instrucionista, construtivista, sociohistórica ou outra).

As escolas que adotam uma abordagem interacionista apresentam importantes vantagens na modelagem de sistemas multiagentes, pois tal abordagem não apenas se preocupa em definir os tipos de sujeitos (agentes) que participam num processo de ensino-aprendizagem, como também 
explicam as características deste processo, através das interações que ocorrem entre os mesmos. Consideram-se escolas interacionistas, tanto o construtivismo cognitivo, baseado nos trabalhos de Piaget, quanto a abordagem baseada na interação social de Vygotski (PASSERINO, 2000; 2000a). O presente trabalho adota a abordagem construtivista, que está sendo implantada no sistema Amplia (SEIXAS et al., 2002), onde o aluno tem um papel ativo no processo de aprendizagem, e o professor um papel de mediador e motivador neste processo, propondo estratégias de reflexão na solução de problemas. Neste contexto, as principais estratégias pedagógicas adotadas são estratégias positivas, voltadas à motivação do aluno e não estratégias negativas, que identificam os erros e os problemas do aluno.

\section{O modelo pedagógico do Amplia}

As características básicas do processo de ensino-aprendizagem do Amplia são:

a) $\mathrm{O}$ processo de ensino-aprendizagem desenvolve-se numa sucessão de etapas de tomada de decisão (diferentes estágios). Cada um destes estágios corresponde a um ciclo de interação entre o Agente Aprendiz e o Agente de Domínio, com a eventual participação do Agente Mediador, para auxiliar na resolução de conflitos.

b) O aluno atualiza seu conhecimento após a análise dos argumentos recebidos durante a execução de cada uma das etapas de tomada de decisão, com o propósito de fundamentar e qualificar suas decisões no próximo estágio. Todas as ações realizadas pelo aluno são registradas pelo Agente Aprendiz. Essas observações apóiam a orientação pedagógica construtivista, onde o conhecimento é construído.

No Amplia, tanto o aluno como o Agente de Domínio participam do processo de ensino/aprendizagem. O conhecimento é assimilado e posteriormente acomodado através de alterações nas estruturas mentais pré-existentes. A reflexão sobre estas alterações origina novas estruturas mentais, que podem sofrer novas acomodações, e assim sucessivamente (BELHOT, 1998). O aluno, ao analisar as observações e sugestões (argumentos) fornecidas pelo Agente Mediador, atualiza seu conhecimento com o propósito de melhorar seu modelo diagnóstico. $\mathrm{O}$ mesmo pode acontecer com o Agente de Domínio, ou seja, partindo do pressuposto de que um estudo de 
casos pode ser modelado de diferentes maneiras, o aluno pode vir a criar um modelo viável, embora não idêntico à solução do especialista.

A interação entre os agentes do sistema está baseada em estratégias pedagógicas que, no Amplia, são de dois tipos: afetivas e cognitivas. Estas estratégias possibilitam o uso intensivo de argumentação para apresentação e esclarecimento de situações, buscando o equilíbrio através de concessões mútuas por parte do Agente Aprendiz e do Agente de Domínio. Na próxima seção descreve-se a dinâmica de interação entre os agentes.

\section{Dinâmica da interação entre os Agentes}

No Amplia, o aluno expressa sua argumentação por meio da modelagem da BN. Bertil Rolf (ROLF; MAGNUSSON, 2002) afirma que a prática e o ensino do raciocínio e da argumentação prestam-se ao uso de esquemas. Compactuamos com essa idéia e, por essa razão, a forma de expressão do aluno ocorre por meio de um editor gráfico, onde os argumentos são formados por nós e pelas ligações entre eles. Rolf classifica os sistemas, que expressam argumentos por meio de estruturas gráficas, em três níveis de software, levando em consideração o cálculo empregado. O sistema Belvedere, por exemplo, não possui cálculo algum, constituindo o primeiro nível e os sistemas Athenas e Reason!Able encontram-se num nível intermediário, contendo alguma designação numérica e regras para filtragem dos melhores argumentos. O Amplia encontra-se no terceiro nível, onde o sistema contém avançada teoria matemática, baseada em inferência bayesiana. Os sistemas mencionados acima, exceto o Amplia, não apresentam um mediador no processo de aprendizagem.

O processo de negociação segue um protocolo de interação/conversação que está esquematizado a seguir:

(1) No estado inicial, o Agente de Domínio apresenta um estudo de caso para o aluno. Neste estado, o Agente Aprendiz apenas toma nota do exemplo e o repassa ao aluno.

(2) No segundo estado, o aluno modela sua hipótese diagnóstica a partir do estudo de casos disponibilizado pelo Agente de Domínio. Ainda no segundo estado, o aluno envia (por meio do Agente Aprendiz) seu modelo ao Agente de Domínio para ser avaliado. Essa avaliação resulta numa classificação dos pontos divergentes do modelo do aluno em relação ao modelo do especialista. A classificação é referente à importância de cada região do modelo 
(trigger, essencial, complementar...). Esta avaliação não é repassada diretamente ao Agente Aprendiz, mas enviada ao Agente Mediador.

(3) No terceiro estado, o Agente Mediador, baseado no resultado da análise do Agente de Domínio e do nível de confiança (declarado pelo aluno) fornecido pelo Agente Aprendiz, escolhe a melhor estratégia pedagógica, acionando a tática adequada à situação em particular.

(4) No quarto estado, o aluno avalia a mensagem recebida do Agente Mediador e procura argumentar sobre os pontos que ele considera importantes, através da alteração de seu modelo. Nesse mesmo estado, o aluno pode também decidir abandonar o processo de aprendizagem (por não se sentir satisfeito, por exemplo).

O processo de negociação do Amplia gira em torno da escolha dinâmica de estratégias. Os parâmetros considerados estão vinculados às crenças do aluno, à avaliação realizada pelo Agente de Domínio e às observações registradas pelo Agente Aprendiz.

Nesse processo de negociação, tanto o aluno como o Agente de Domínio tem a possibilidade de abandonar a interação. Entretanto, o Agente de Domínio somente abandona o processo de negociação quando o aluno apresenta uma solução cuja performance seja equivalente ou melhor que a do seu modelo. O Agente de Domínio pode vir a aceitar a modelagem do aluno, embora não corresponda exatamente ao seu modelo, ou seja, caso os argumentos realizados pelo aluno solucionem o problema do estudo de caso apresentado ao aluno.

\section{Exemplo hipotético}

A seguir é apresentado um exemplo hipotético do uso do Amplia.

Após identificar o aluno, o Agente Aprendiz seleciona e apresenta um estudo de caso disponível na Base de Dados do Agente de Domínio. Se o aluno manifestar concordância para iniciar os estudos, o Agente de Domínio apresenta uma lista com todos os nós, relacionados ou não com o caso em questão, e o aluno inicia o desenvolvimento de uma BN, através do editor gráfico Seamed (FLORES et al., 2001), selecionando os nós que considerar e relacionando-os entre si.

O aluno pode elaborar as partes qualitativas e quantitativas do modelo, identificando as variáveis que são "sintomas" e os "diagnósticos" apresen- 
tadas no caso, estimando a distribuição de probabilidade condicional inicial do conjunto de variáveis selecionadas.

A seguir, o Agente Aprendiz envia a parte qualitativa da rede do aluno, juntamente com a distribuição de probabilidades a priori e a posteriori, para o Agente de Domínio. Enquanto isso, o Agente Mediador solicita que o aluno informe o grau de confiança que este tem em seu modelo (alta, média ou baixa). O Agente de Domínio inicia a comparação tanto do relacionamento causal como das probabilidades com o modelo construído pelo especialista do domínio e envia o resultado ao Agente Mediador. Este resultado classifica a rede do aluno em inviável, incorreta, incompleta, viável ou completa.

Finalmente, considerando as informações do modelo do aluno, o resultado da comparação entre as redes do aluno e do especialista e o grau de confiança declarado pelo aluno, o Agente Mediador seleciona uma estratégia para negociar com o aluno, de modo a estimulá-lo a esclarecer suas dúvidas e a rever seu modelo de rede, para que este se torne cada vez mais completo e eficiente.

\section{Conclusão}

A possibilidade desta linha de financiamento, criada pela Fapergs e pelo $\mathrm{CNPq}$, permitiu a continuidade da troca de conhecimentos e da coparticipação na formação de recursos humanos entre os grupos da UFRGS e da UCS.

O sistema desenvolvido tem resultado em benefício para os alunos de residência médica e para os clínicos gerais de regiões remotas, num primeiro momento no RS, em SC e em BSB. Isso graças ao fato de que o sistema pode operar, tanto como sistema de apoio ao diagnóstico, como também de treinamento nas áreas em que existem redes de conhecimento disponíveis no banco de conhecimentos do sistema.

O processo de negociação aqui envolvido é diferente, em diversos aspectos, dos sistemas de negociação baseados na Teoria dos Jogos e das Teorias de Mercado, pois se trata de uma negociação de cunho pedagógico. Estamos interessados no desenvolvimento de agentes autônomos capazes de auxiliar na aprendizagem, sendo esta baseada na experiência e na 
melhoria incremental do comportamento do agente. A negociação na área da educação está diretamente ligada à forma pedagógica de como levar um aluno a desenvolver seu raciocínio diagnóstico, aperfeiçoando a sua capacidade de aprendizagem.

A negociação pedagógica usa o Agente Mediador para resolver impasses que possam ocorrer na interação entre o Agente de Domínio e o aluno (e o seu correspondente Agente Aprendiz). A negociação no Amplia acontece em níveis de tolerância de impasse, cabendo ao Agente Mediador a escolha da tática a ser utilizada. O processo de negociação ocorre em rounds (ciclos) de decisão, cujo ciclo é iniciado com a submissão do modelo do aluno para avaliação.

A negociação se fundamenta no próprio processo de aprendizagem do aluno. Partindo do pressuposto de que a sua capacidade de absorver/considerar todos os achados de uma modelagem diagnóstica seja afetada pela sua própria experiência como profissional, o processo de negociação intuitivamente permite, por meio da argumentação, modificar crenças do outro agente.

A negociação pedagógica é um processo de resolução de conflitos que ocorrem entre os agentes participantes de um processo de ensinoaprendizagem. Dentro da abordagem apresentada, este conflito ocorre justamente quando acontece uma quebra de confiança de um agente em relação ao outro. Na prática, isto se traduz no não atendimento de uma expectativa de comportamento ou desempenho que um agente tem em relação ao outro.

Por outro lado, num processo de negociação deve-se considerar o espaço de confronto, onde há várias possibilidades para a solução de um impasse. Isso ocorre durante a modelagem de uma hipótese diagnóstica. Ou seja, um diagnóstico pode ser modelado por diferentes formas (relação causal).

$\mathrm{Na}$ prática, e de acordo com a abordagem exposta, o resultado final esperado da negociação é que as expectativas do aluno em relação ao professor e do professor em relação ao aluno se confirmem e que, portanto, um alto grau de confiança seja estabelecido nas capacidades um do outro.

Os grupos de pesquisa envolvidos capacitaram-se no uso e no desenvolvimento (uma vez que o sistema para a representação de conhecimento através de redes probabilísticas é aberto) de ferramentas para a aquisição e a representação do conhecimento em redes probabilísticas. As equipes também adquiriram o conhecimento necessário para trabalhar com a Plataforma multiagentes Fipa. 


\section{REFERÊNCIAS}

ANDRADE, A. F. et al. A Computational Model of Distance Learning Based on Vygotsky's Socio-Cultural Approach. In: MULTI-AGENT ARCHITECTURES SUPPORTING DISTRIBUTED LEARNING IN A WIRED AND WIRELESS FUTURE WORKSHOP, San Antonio, May 2001. Proceedings... San Antonio, 2001.

BELHOT, R. V. Searching for New Ways of teaching. In: INTERNATIONAL CONFERENCE OF ENGINEERING EDUCATION - ICEE -, 1998, Rio de Janeiro. Disponível em: 〈http://www.ime.eb.br/icee98/pappers/232.pdf>

FERBER, J.; GUTKNECHT, O. A meta-model for the analysis and design of organizations in multi-agent systems. In: INTERNATIONAL CONFERENCE ON MULTI-AGENT SYSTEMS - ICMAS'98 -, IEEE COMPUTER SOCIETY PRESS, 3., July 1998, Paris. Proceedings... Paris, 1998. p. 128-135.

FLORES, C. D. et al. Una experiencia en el uso de redes probabilisticas para el diagnóstico médico - una experiencia brasileña.Informátic@ Médica, Argentina, v. 8, p. 25-29, 2001.

. et al. Processo de negociação em um ambiente de aprendizagem inteligente. In: CBCOMP2002 - WORKSHOP DE INFORMÁTICA APLICADA À SAÚDE, 2. 2002, Itajaí. Anais... Itajaí, 2002.

JENSEN, F. V.; OLSEN, K. G.; ANDERSEN, S. K. AnAlgebra of Bayesian Belief Universes for Knowledge-Based Systems. Networks. New York: John Wiley \& Sons Inc., 1990. v. 20, p. 637-659.

FIPA-OS. Nortel Networks Harlow Laboratories. Disponível em: <http:// www.nortelnetworks.com/products/announcements/fipa/software.html>Acesso em: 04 nov. 2001.

PASSERINO, L. M. Interaction Sociale dans l'environment telematiques d'aprentissage. In: COLLOQUE CONSTRUCTIVISMES USAGES ET PERSPECTIVES EM ÉDUCATION, Genebra, 2000.

PASSERINO, L. M.; SANTAROSA, L. C. Uma visão sócio-histórica da interação dentro de ambientes computacionais. In: CONGRESSO IBERO-AMERICANO DE INFORMÁTICA NA EDUCAÇÃO - RIBIE, 5., Vinã del Mar, Chile, 2000.

RIBEIRO, A. M.; DEMAZEAU, Y. Um modelo de interação baseado em mensagens ativas. In: CONFERÊNCIA LATINO-AMERICANA DE INFORMÁTICA, 27., 2428 set. 2001, Mérida, Venezuela. Anais... Mérida, 2001.

RIBEIRO, A. M.; CASA, M. E. Construção de uma plataforma multiagentes para o ambiente de aprendizagem ILENA. In: WORKSHOP DE AMBIENTES DE APRENDIZAGEM BASEADOS EM AGENTES, 3., 22 nov. 2001, Vitória. Anais... Vitória, 2001. 
ROLF, B.; MAGNUSSON, C. Developing the art of argumentation, a software approach. INTERNATIONAL CONFERENCE ON ARGUMENTATION - ISSA-, 5., June 2002, Amsterdam. Proceedings... Amsterdam: University of Amsterdam, 2002.

SEIXAS, L. et al. An Architecture for an Intelligent Learning Environment with a Constructivist Approach. In: INTELLIGENT TUTORING SYSTEMS - ITS' -, 2002, San Sebastian. Proceedings... San Sebastian, 2002. v. 1, p. 8-15.

VICARI, R. M. et al. A Multi-Agent Intelligent Environment for Medical Knowledge. Artificial Intelligence in Medicine. Elsevier Science, v. 27, n. 3, p. 335-366, Mar. 2003.

Texto recebido em 25 maio 2003 Texto aprovado em 05 set. 2003 